\title{
Differences in temporal appearance associated with activity in the chromatic and achromatic systems
}

\author{
STEVEN H. SCHWARTZ and MICHAEL S. LOOP \\ University of Alabama, Birmingham, Alabama
}

The temporal appearances of spatially coincident increments that activate the chromatic or achromatic system are different. Whereas near-threshold stimuli that are detected by the chromatic system appear to come on gradually, increments detected by the achromatic system appear to flash or step on. This difference in perception is consistent with previous reports that show differences in the temporal properties of the two systems.

It is generally assumed that the human visual system processes color information along at least two distinct channels or systems. The chromatic system is postulated to code hue information, and the achromatic system, to code luminance cues. Support for this model of color vision comes from both electrophysiological and psychophysical investigations (Boynton, 1979).

One obvious feature that characterizes the operation of these two systems in isolation concerns the percept associated with activation of the system. It is reasonable to assume that stimulation of the isolated chromatic system will result in a colored percept. In contrast, activation of the isolated achromatic system should produce an achromatic percept. Consistent with this model, King-Smith and Carden (1976) demonstrated that threshold spectral sensitivity functions characterizing the chromatic system are associated with a color percept and that spectral sensitivity functions for the achromatic system are associated with a colorless percept.

It would be surprising if the only difference in the percepts associated with these isolated channels is the color/colorless distinction. Additional psychophysical studies suggest that these systems show different temporal properties (Bowen, Lindsey, \& Smith, 1977; Kelly \& van Norren, 1977; King-Smith \& Carden, 1976; Nissen \& Pokorny, 1977; Regan \& Tyler, 1971; Schwartz \& Loop, 1982). Presumably, these additional distinguishing properties may influence the threshold percepts associated with these systems.

This work was supported by National Institutes of Health Grants EY 03303-04, EY 03039-01, and RR 05807. We thank Caroline Dunn for secretarial assistance in the preparation of the manuscript. The authors' complete mailing address is: Department of Physiological Optics, School of Optometry/The Medical Center, University of Alabama in Birmingham, Birmingham, Alabama 35294.
In the course of determining reaction time (RT) distributions to long-duration near-threshold stimuli, the authors noted an obvious difference in the temporal appearance of stimuli that activated either the chromatic or the achromatic system. Stimuli for the achromatic system appeared as flashes or step changes at detection, whereas stimuli for the chromatic system had slow or gradual onsets. The difference was striking. Therefore, this phenomenon was further investigated, with naive subjects.

\section{EXPERIMENT 1}

\section{Method}

Subjects. Three (two females-C.G., W.M.; one male-B.P.) students, in their mid-20s, participated in the study. All had had previous experience in psychophysical investigations but were naive with respect to the current study. All demonstrated normal color vision on the Dvorine pseudoisochromatic plate test.

Stimuli. The stimuli and background were presented in Maxwellian view, and both were $4 \mathrm{deg}$ in diameter. Stimulus duration was $1,000 \mathrm{msec}$, and temporal presentation was squarewave onset and of fset.

Stimuli predicted to reflect detection by the chromatic or the achromatic system were obtained with conditions comparable to those of King-Smith and Carden (1976). The background was white and had a retinal illuminance of $1,000 \mathrm{Td}$. The chromatic system was studied with increments of $680 \mathrm{~nm}$ and $620 \mathrm{~nm}$, and white increments were used to study the achromatic system.

Apparatus and Procedure. RT distributions were determined in response to 1,000 -msec near-threshold intensity increments. The subject depressed a telegraph key with the right hand, and solid state programming modules presented the stimulus following a random pre-time period. The subject's task was to release the key as soon as any change in the stimulus field was noted. RT was recorded to the nearest millisecond. The subject had only one opportunity to respond to each stimulus, and stimuli were not response terminated.

After responding to each stimulus, the subject used a pushbutton device held in the left hand to signal the temporal percept associated with the stimulus's detection. The subject characterized the temporal appearance of the stimulus by placing it into one of the following three categories: (1) the stimulus appeared as a flash or step change, (2) the stimulus appeared as 
a slow or gradual change, and (3) the stimulus did not fall into either of the first two categories.

During a single experimental condition, either 100 or 150 trials were conducted under a single set of experimental conditions. This allowed a RT histogram to be obtained for each experimental session.

\section{Results}

The data are represented in the form of RT histograms. For each RT, the subject's percept is known and is indicated on the histograms by appropriate shading. Black represents stimuli seen as flashes, and white, as slow changes. Fewer than $2 \%$ of the stimuli fell into Category 3 , and this category is considered no further. Figure 1 displays RT distributions for stimuli predicted to isolate the chromatic system. The general shape of the histograms is similar to shapes previously reported for the chromatic system (Schwartz \& Loop, 1982). The percept associated with detection of these stimuli is that of a slow change; the subjects reported that these stimuli came on gradually.

Figure 2 shows the RT distributions elicited by the white increments. The form of these distributions is similar to forms previously reported for the achromatic system: detection is tightly associated with stimulus onset or offset (Schwartz \& Loop, 1982). In contrast to Figure 1, the percept

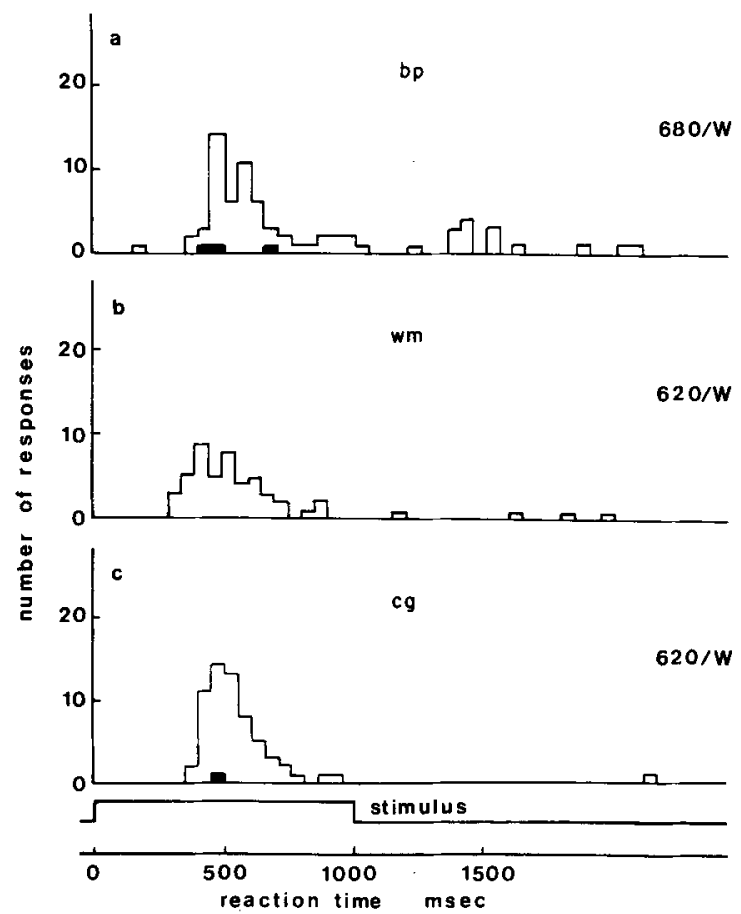

Figure 1. RT distributions, with the associated percepts displayed on the histograms, for stimuli predicted to reflect activity in the chromatic system. Each histogram represents data for a different subject. Stimulus conditions are indicated at the right side of each histogram. Bin width is 50 msec. Open areas represent stimuli seen as slow changes; dark areas represent stimuli perceived as fast changes. Detection rates were (a) $60.0 \%$, (b) $70.8 \%$, and (c) $78.8 \%$.

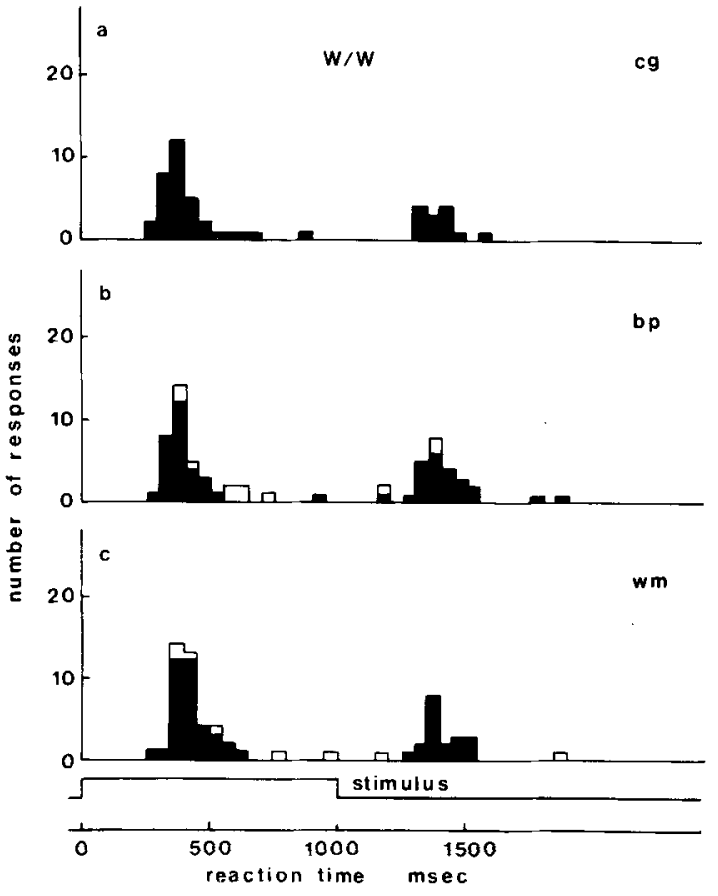

Figure 2. RT distributions, with associated percepts, for stimuli predicted to reflect activity in the achromatic system. Both the stimulus and the background were white. Each histogram represents one subject. The code for displaying percepts is the same as that used in Figure 1. Detection rates were (a) $60.3 \%$, (b) $55.6 \%$, and (c) $81.3 \%$.

generally associated with these stimuli is that of a flash or step change. Only a few of these stimuli appeared as slow changes. Therefore, the temporal percepts associated with activity in the achromatic and chromatic systems are clearly different. Note that the detection rates associated with the two samples of histograms are approximately equal (nearthreshold values), thus eliminating differences in detectability as a factor in producing the different temporal percepts.

\section{EXPERIMENT 2}

Increasing background adaptation levels results in increased sensitivity of the chromatic system relative to the achromatic system (King-Smith \& Carden, 1976). This results in a situation in which threshold $580-\mathrm{nm}$ increments on relatively dim backgrounds are detected mostly by the achromatic system and on brighter backgrounds, mostly by the chromatic system. This shift from detection by the achromatic system on dim backgrounds to detection by the chromatic system on brighter backgrounds should be reflected in the temporal perceptions associated with these stimulus conditions. The prediction is, therefore, that, on a relatively dim background, 580-nm increments will mostly appear as flashes but that, on a brighter background, they will appear as slow temporal changes. 

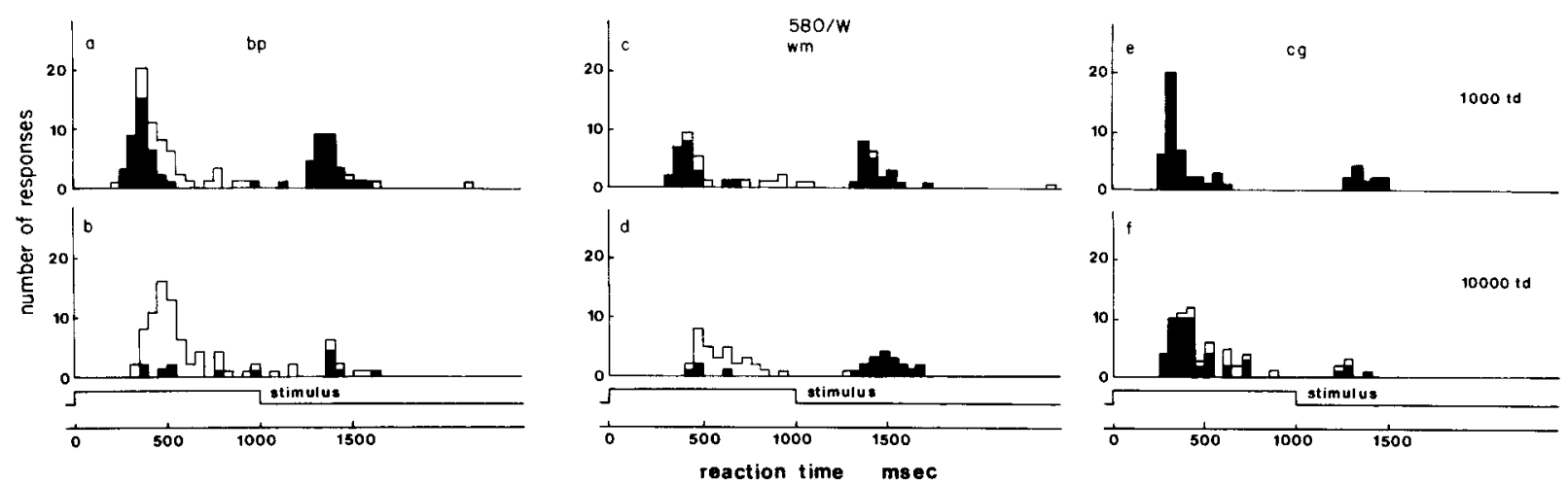

Figure 3. RT histograms for 580-nm increments on both a 1,000-Td and 10,000-Td white background. Each column represents a different subject, and each row represents a different background luminance. Detection rates were (a) $86.2 \%$, (b) $73.7 \%$, (c) $75.0 \%$, (d) $65.4 \%$, (e) $72.6 \%$, and (f) $88.6 \%$.

\section{Methods}

Subjects. The three subjects of Experiment 1 participated in this experiment.

Stimull. The increment was $580 \mathrm{~nm}$, and the background was either 1,000 or $10,000 \mathrm{Td}$. Duration was $1,000 \mathrm{msec}$. King-Smith and Carden's (1976) data suggest that with a 1,000-Td background, the two systems' sensitivities will be similar, but with the achromatic system showing slightly greater sensitivity.

\section{Results}

With a 1,000-Td background, Subject C.G. reported that all of the stimuli appeared as flashes (Figure 3). For Subjects B.P. and W.M., some of the increments resulted in a percept of a slow change, but most were seen as flashes. The data of KingSmith and Carden (1976) suggest that with a 1,000Td background, the sensitivities of the two systems are close; therefore, the appearance of some of these stimuli as slow changes is not surprising.

At the brighter adaptation level $(10,000 \mathrm{Td})$, the temporal appearance of the stimuli was clearly different. This is most obvious for Subjects B.P. and W.M.; many of the 580-nm increments were now perceived as slow temporal changes. With Subject C.G., the phenomena is not as obvious, but at this brighter adaptation level some increments were seen as slow changes, while with the dimmer background all were seen as flashes.

\section{DISCUSSION}

Previous research has suggested that threshold stimulation of the isolated chromatic system results in a colored percept, whereas stimulation of the achromatic system results in a colorless percept (King-Smith \& Carden, 1976). This report demonstrates another difference in the percepts associated with activity in these two systems. Near-threshold stimulation of the chromatic system, with temporal square-wave stimuli, results in the perception of a slow or gradual temporal change. In contrast, the achromatic system is associated with the perception of a flash or step change.
This difference in temporal perception for the two systems is consistent with both physiological (Gouras, 1968; Schiller \& Malpeli, 1978) and psychophysical (Bowen, Lindsey, \& Smith, 1977; Kelly \& van Norren, 1977) reports that suggest these systems display different temporal sensitivities. For example, the slow temporal change associated with the chromatic system is compatible with the broad low-frequency peak of the chromatic system's temporal modulation transfer function (TMTF) (Kelly \& van Norren, 1977). Also, the achromatic system's TMTF's narrow peak at relatively high frequencies (Kelly \& van Norren, 1977) is consistent with the appearance as a flash or step change.

\section{REFERENCES}

Bowen, R. W., Lindsey, D. T., \& Smith, V. C. Chromatic two-pulse resolution with and without luminance transients. Journal of the Optical Society of America, 1977, 67, 1501-1507.

Boynton, R. M. Human color vision. New York: Holt, Rinehart \& Winston, 1979.

GourAs, P. Identification of cone mechanisms in monkey ganglion cells. Journal of Physiology (London), 1968, 199, 533-547.

Kelly, D. H., \& van NorRen, D. Two-band model of heterochromatic flicker. Journal of the Optical Society of America, 1977, 67, 1081-1091.

King-Smith, P. E., \& Carden, D. Luminance and opponentcolor contributions to visual detection and adaptation and to temporal and spatial integration. Journal of the Optical Society of America, 1976, 66, 709-717.

Nissen, M. J., \& Pororny, J. Wavelength effects on simple reaction time. Perception \& Psychophysics, 1977, 22, 457-462.

REgan, D., \& TYLER, C. W. Temporal summation and its limit for wavelength changes: An analog of Bloch's law for color vision. Journal of the Optical Society of America, 1971, 61, 1414-1421.

Schiller, P. H., \& Malpeli, J. G. Functional specificity of lateral geniculate nucleus laminae of the rhesus monkey. Journal of Neurophysiology, 1978, 41, 788-797.

Schwartz, S. H., \& LOOP, M. S. Evidence for transient luminance and quasi-sustained color mechanisms. Vision Research, 1982, 22, 445-447.

(Manuscript received November 5, 1982;

revision accepted for publication December 15, 1982.) 